\title{
Modulation of membrane structure and function by hydrophobic mismatch between proteins and lipids
}

\author{
J. Antoinette Killian ${ }^{1}$, Maurits R.R. de Planque ${ }^{1}$, Patrick C.A. van der $\mathrm{Wel}^{2}$, Irene \\ Salemink ${ }^{1}$, Ben de Kruijff ${ }^{1}$, Denise V. Greathouse ${ }^{2}$ and Roger E. Koeppe II 2 \\ ${ }^{1}$ Dept. Biochemistry of Membranes, Utrecht University (NL) and 2 Dept. of Chemistry \\ and Biochemistry, University of Arkansas (USA)
}

The structure and function of biological membranes can be expected to be sensitive to
the extent of hydrophobic matching between the length of the membrane-spanning part
of intrinsic membrane proteins and the hydrophobic thickness of the lipid bilayer. To
gain insight into the consequences of hydrophobic mismatch on molecular level, we
have carried out systematic studies on well-defined peptide/lipid complexes, using
artifical transmembrane peptides, anchored to the membrane by tryptophan residues. It
is shown that hydrophobic mismatch can result in a dramatic change in lipid
organization, and that the presence of interfacially localized aromatic amino acid
residues is important for determining the exact consequences of hydrophobic mismatch.

\section{INTRODUCTION}

The basic building blocks of biological membranes are lipids and proteins. The proteins are embedded with their hydrophobic parts in contact with the acyl chain region of the lipid bilayer. In recent years it has become increasingly clear that the structural and functional properties of membranes are highly sensitive to the precise way in which the proteins are embedded in the lipid bilayer. In particular, the extent to which the hydrophobic thickness of the lipid bilayer matches that of the membrane-spanning part of the proteins may be an important parameter in determining membrane structure and function. This is suggested by theoretical studies [1], by reconstitution studies of membrane proteins in lipid bilayers of varying thickness $[2,3]$, and by the observed correlation between the length of the membrane-spanning segment of proteins in different biological membranes and the hydrophobic thickness of these membranes [4].

The importance of hydrophobic matching can be easily understood on the basis that a mismatch would result in exposure of hydrophobic surfaces of either the protein or the lipids to an aqueous environment. Both proteins and lipids may react to prevent this, and they can do so in a number of different ways, as illustrated in Figure 1. Proteins could aggregate, tilt to change their effective hydrophobic length, adapt their secondary structure, or adopt another conformation. The lipids can react by either stretching or disordering their acyl chains, or, more rigourously, by adopting a non-bilayer phase, as observed for the transmembrane polypeptide gramicidin [5] and for various signal peptides [6]. When mixtures of lipids are present, the protein may become surrounded preferentially by the lipids with the most optimal hydrophobic matching [7-9].

Little is known about which of these counteractive moves will be preferred under a particular condition. Yet such rearrangements can have important implications for membrane structure and function. Protein function for instance could be regulated by a tendency to self-associate of helices with a similar mismatch in length, leading to specific protein-protein interactions or helping to obtain a correct assembly of multi membrane-spanning proteins. Tilting of helices will affect interactions between side chains in adjacent helices, while also any conformational changes may influence or regulate protein function. Effects of mismatch on lipid structure and organization may have implications for other aspects of membrane functioning, for instance by interfering with processes that require the local and transient formation of non bilayer structures [10], or with processes that are sensitive to lipid packing. 


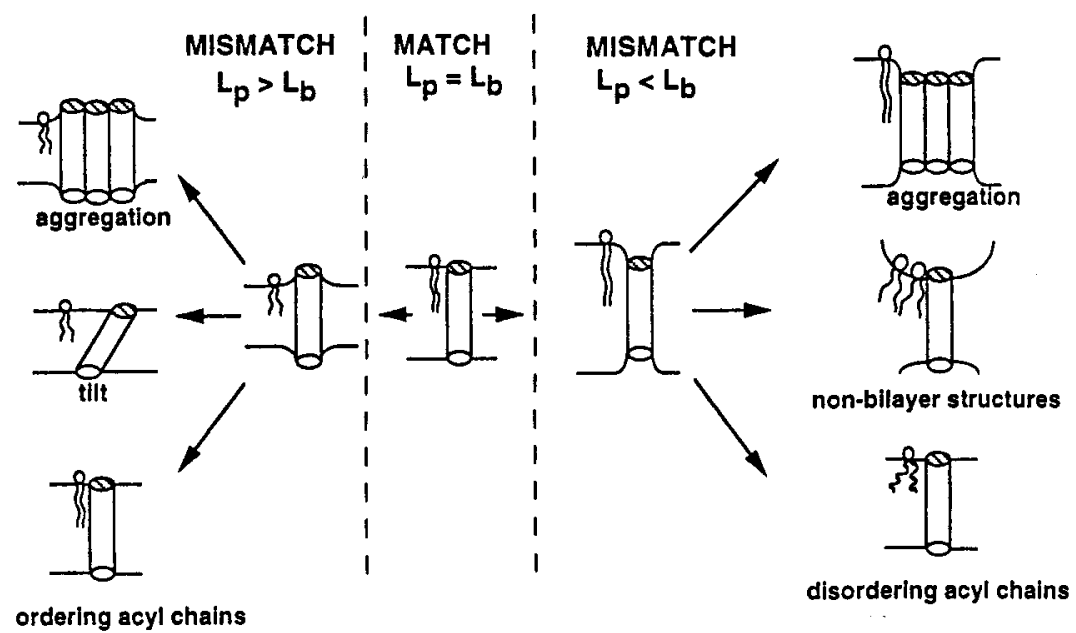

Fig.1 Schematic representation of some possible consequences of hydrophobic mismatch ( $\mathrm{L}_{\mathrm{b}}$ and $\mathrm{L}_{\mathrm{p}}$ are hydrophobic length of bilayer and protein, respectively)

The consequences of hydrophobic mismatch will depend on properties of the lipids as well as on specific properties of the proteins. To gain insight into what factors determine the precise consequences of hydrophobic mismatch, it is necessary to carry out systematic studies on well-defined protein-lipid complexes.

\section{Choice of lipids}

Membrane lipids can be roughly divided into two types of lipids: those, that when hydrated in excess water under physiological conditions of $\mathrm{pH}$, temperature and salt concentration, spontaneously organize in bilayers, and those that under the same conditions prefer organization in non-bilayer structures with a concave surface curvature, such as the reversed hexagonal (HII) phase. A rationale for the different behaviour of isolated membrane lipids, originally proposed by Israelachvili and coworkers [11], is depicted in Figure 2. According to this concept, bilayer preferring lipids have an overall nearly cylindrical shape, in which the surface area of the headgroup region approximately matches that of the hydrocarbon area. When the area of the headgroup region is small compared to that of the acyl chains, the lipids will tend to form non-bilayer structures with a net negative surface curvature (type II structures).

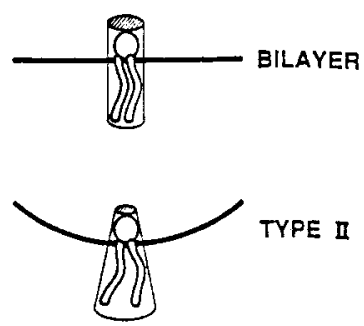

Fig. 2 Shape-structure relationship of lipids, adapted from Cullis \& De Kruijff [12]

Non-bilayer lipids are abundant in most membranes, where there is ample evidence that their specific properties play an important structural and functional role [13]. However, in biological membranes these lipids will always be mixed with bilayer preferring lipids, leading to an overall bilayer organization which is essential for the barrier function of these membranes. For this reason we chose to start our experiments on the effects of hydrophobic mismatch by using phosphatidylcholine (Figure 3 ), a lipid that on its own will not organize in non-bilayer structures, but will form a stable bilayer. This lipid is abundant in many membranes and has the advantage that it carries no net charge, avoiding complications due to electrostatic interactions. 


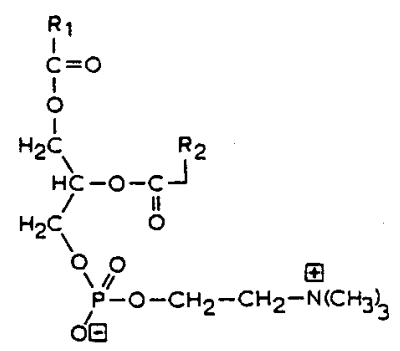

Fig.3 Structure of diacylphosphatidylcholine (PC)

\title{
Choice of peptides
}

Since multi membrane-spanning proteins are rather complex, we chose to use model transmembrane peptides for systematic studies on the consequences of hydrophobic mismatch. A family of peptides was designed (Table I) with a hydrophobic core of leucine, alternating with alanine to promote solubility of the peptide. Both leucine and alanine have a high propensity to form $\alpha$-helical structures. The peptides are completely uncharged, hydrophobic, and contain tryptophans at both ends. This mimicks the situation found for the membrane-spanning part of type I single membrane-spanning proteins [14] as well as of multi membrane-spanning proteins, in which tryptophans are highly enriched at the membrane/water interface [e.g. 15,16]. Since tryptophans have a high affinity for the interfacial region [17], it is likely that they play a role as membrane anchor. The peptides, named WALP based on their amino acid composition, have their $\mathrm{N}$ - and $\mathrm{C}$-termini blocked, the latter with a chemically accessible ethanolamine group to allow attachment of (fluorescent or spin-labeled) reporter groups. The peptides contain no other reactive groups.

\section{TABLE 1: Structure of WALP peptides}

\author{
WALP16: HCO-AWWLALALALALAWWA- $\mathrm{NHCH}_{2} \mathrm{CH}_{2} \mathrm{OH}$ \\ WALP17: HCO-AWWLALALALALALWWA- $\mathrm{NHCH}_{2} \mathrm{CH}_{2} \mathrm{OH}$ \\ WALP19: HCO-AWWLALALALALALALWWA-N $\mathrm{NCH}_{2} \mathrm{CH}_{2} \mathrm{OH}$
}

By circular dichroism studies of the WALP peptides in diacylphosphatidylcholine bilayers, it could be established that these peptides indeed adopt a transmembrane $\alpha$-helical structure [18].

\section{EFFECTS OF MISMATCH ON LIPID ORGANIZATION}

First it was investigated whether the peptides affect lipid phase behavior as a consequence of mismatch. ${ }^{31}$ P NMR was used as a convenient technique to discriminate between organization of phospholipids in bilayer and in non-bilayer structures (Figure 4). When lipids are organized in a bilayer, fast axial reorientation of the lipids about their long axis results in partial averaging of the chemical shift anisotropy, giving rise to characteristic spectra with a low field shoulder and a high field peak $[12,19]$. The nonbilayer phase that is preferentially adopted by isolated non-bilayer lipids is the hexagonal HII phase. In this phase the phospholipids are arranged in long tubes, with their headgroups surrounding an aqueous channel, which allows the lipids to express their preference for a curved lipid/water interface. In addition to fast axial reorientation, the lipids now also undergo fast diffusion about the tubes of the HII phase, resulting in a $31_{\mathrm{P}}$ NMR spectrum with a reversed asymmetry, in which the chemical shift anisotropy is further reduced by a factor of two [12]. Another phase, that can be adopted by mixtures of bilayer and non-bilayer lipids, is the cubic phase. This phase consists of interwoven networks of aqueous channels and highly curved lipid bilayers [20]. In phase diagrams such cubic phases usually can be found inbetween the phase boundaries for a bilayer and for a hexagonal HII phase. The lipids in these phases undergo complete motional averaging, leading to an isotropic $31 \mathrm{P}$ NMR signal. It should be noted, however, that other arrangements of lipids are possible which result in complete averaging of the chemical shift anisotropy and therefore give rise to an isotropic 31 P NMR signal. Examples are micelles and small vesicles. 


\section{LIPID PHASES}
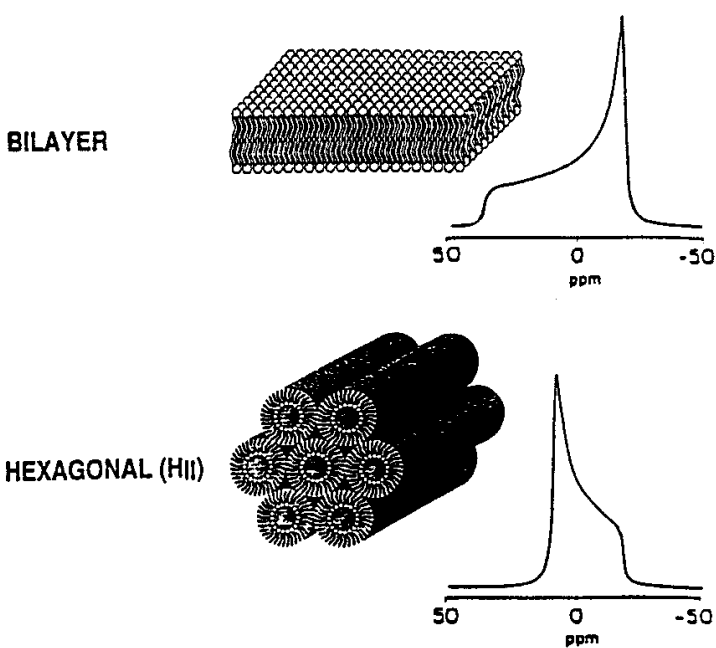

CUBIC

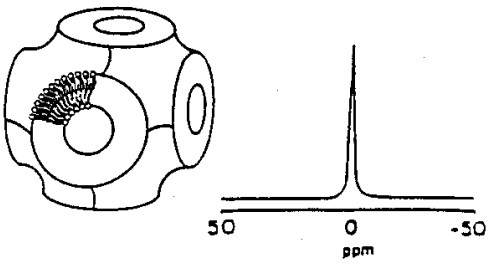

Fig.4 Lipid phases with corresponding ${ }^{31} \mathrm{P}$ NMR spectra

Figure 5 shows ${ }^{31}$ P NMR spectra of dispersions of saturated phosphatidylcholines in the absence and presence of WALP17 and WALP19. In the absence of peptide all lipids are in a bilayer organization, as expected. Upon incorporation of WALP17 in di-C14:0-PC a bilayer organization is maintained, but in diC16:0-PC in addition an isotropic component is observed, demonstrating destabilization of the lipid bilayer and most likely representing a cubic-like phase. Upon further increasing the acyl chain length to $18 \mathrm{C}$-atoms, a different second spectral component is observed, typical for the presence of lipids in an inverted hexagonal (HII) phase. The relative amount of lipids present in the HII phase increases with the acyl chain length, as evident from the higher intensity of the low field peak in di-C20:0-PC. In the presence of WALP 19 almost identical NMR spectra are obtained as for WALP17, but at an acyl chain length of 2 C-atoms longer. Since WALP19 is about $3 \AA$ longer than WALP17, and since an increase by two methylenes in the acyl chains will result in an increase in bilayer thickness of about the same amount (see Table 2), there can be no other conclusion than that it is the extent of hydrophobic mismatch that is responsible for the ability of these peptides to induce non bilayer structures.

TABLE 2: Length of the peptides and hydrophobic thickness of the lipid bilayers

\begin{tabular}{lcc} 
Peptide & \multicolumn{1}{c}{ Length $^{\mathrm{a}}(\AA)$} & Lipid \\
WALP16: & $25.5 \mathrm{Di}-\mathrm{C} 14: 0-\mathrm{PC}$ & 22.8 \\
WALP17: & $27.0 \mathrm{Di}-\mathrm{C} 16: 0-\mathrm{PC}$ & 26.3 \\
WALP19: & $30.0 \mathrm{Di}-\mathrm{C18}: 0-\mathrm{PC}$ & 29.8 \\
& Di-C20:0-PC & 32.8
\end{tabular}

a It is assumed that the peptide is present as an $\alpha$-helix and that each amino acid has a length of $1.5 \AA$, with the C-terminal ethanolamine included as the length of an additional amino acid. $b$ Thicknesses of the hydrophobic part of lipid bilayers in the liquid crystalline phase were calculated according to ref. 21 


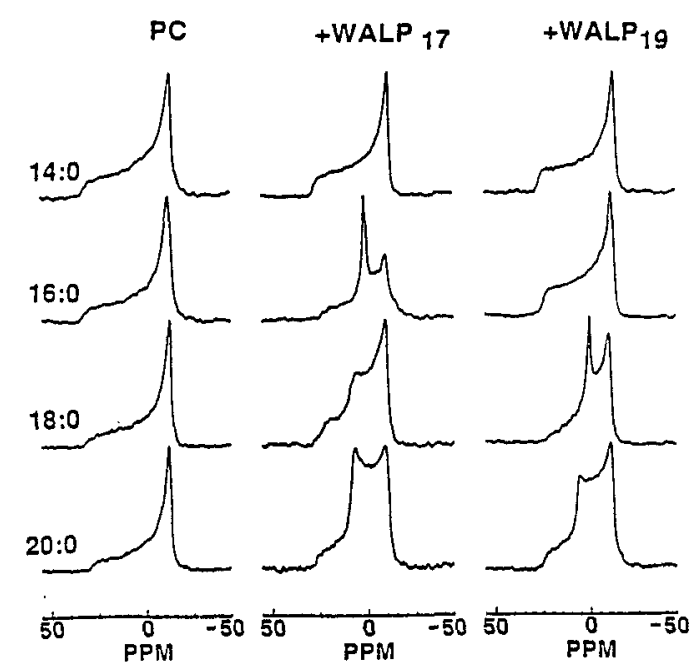

Fig. $53 l_{\mathrm{P}}$ NMR spectra of dispersions of phosphatidylcholines with different acyl chain length in the absence and presence of WALP17 and WALP19. The peptide/lipid molar ratio in these samples was $1 / 10$. Spectra were recorded at temperatures just above the gel to liquid-crystalline phase transition. For details see ref. 18

Similar experiments were performed with unsaturated lipids, and making use of an additional length analog of the peptides, WALP16. The results were very much in line with those described above for the saturated lipids. In fact, when for each sample the hydrophobic thickness of the lipid bilayer was subtracted from the length of the incorporated peptide, and the extents of mismatch were plotted in order of positive mismatch to negative mismatch (Figure 6), it was found that sharp 'mismatch boundaries' exist between the formation of a bilayer and an isotropic phase, and between the formation of an isotropic and an $\mathrm{HII}_{\text {II }}$ phase.

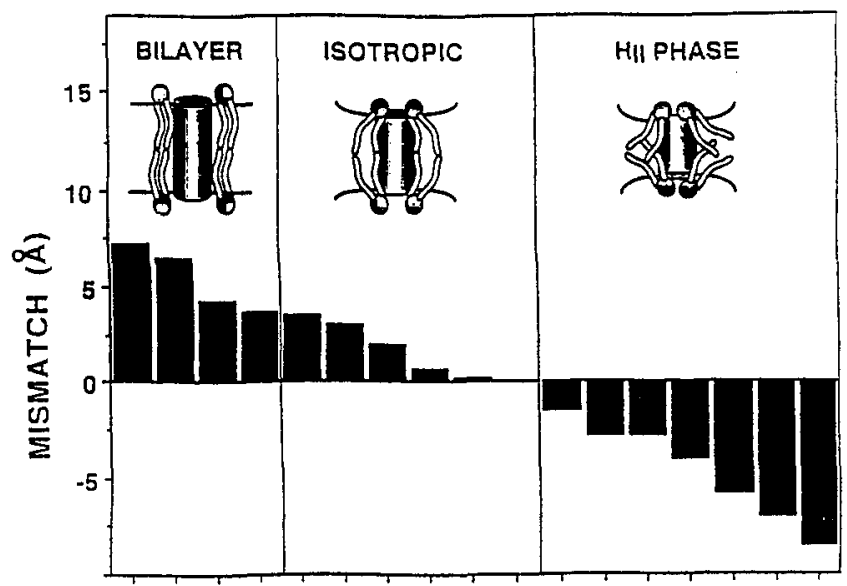

Fig. 6 Phase preference as a function of mismatch for different samples of WALP peptides with saturated and unsaturated lipids. Each bar represents the outcome of one sample. The areas marked isotropic and $H_{I I}$ phase indicate spectra of which part or all of the lipids are present in an isotropic or HII phase, respectively. For details see ref. 18

\section{IMPORTANCE OF TRYPTOPHAN RESIDUES}

The formation of non-bilayer structures in phosphatidylcholine bilayers by transmembrane peptides is a striking result, because up until now this behavior had not been observed for any other peptide, except for the channel forming peptide gramicidin A [22, 23], which also has been frequently used as model for the membrane-spanning part of intrinsic membrane proteins. Even the uncharged, hydrophobic $\alpha$-helical 
polypeptide Boc-(L-Ala-Aib-L-Ala-Aib-L-Ala)3-OMe (where Aib is aminoisobutyric acid), called P15. which is slightly shorter than gramicidin, was found to be unable to affect lipid organization under the same conditions [24]. Comparison of the structures of gramicidin A and WALP 17 (Figure 7) shows that they not only have about the same length, they also both have interfacially localized tryptophans. We propose that it is these residues that are responsible for the induction of non-bilayer structures in phosphatidylcholine systems as a consequence of hydrophobic mismatch.

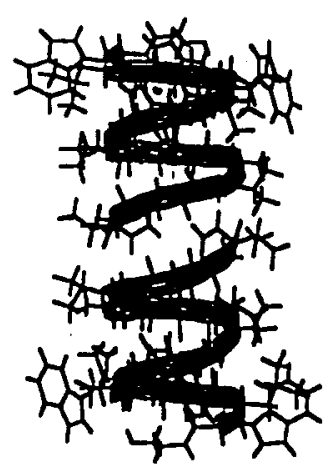

Gramicidin

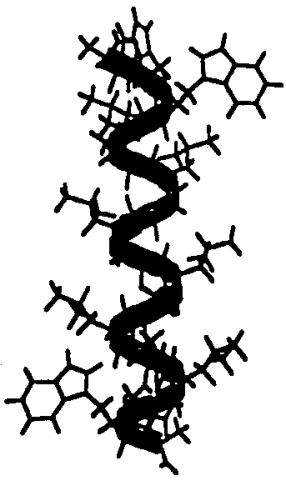

WALP-17

Fig. 7 Structures of gramicidin A in its channel conformation and WALPI7

Evidence for an important role of tryptophans in peptide/lipid interactions was obtained by chemical modification of these residues. Replacement of the indole proton by a formylgroup led to a complete loss of the ability of gramicidin to induce HII phase formation (Figure 8). Preliminary experiments with the WALP peptides indicated a similar loss of the ability to induce non-bilayer structures upon formylation of the tryptophan residues, supporting our suggestion that these residues play an important role in determining the consequences of hydrophobic mismatch for lipid organization.

GRAM. A - L - Trp - D - Leu - L - Trp - D - Leu - L - Irp - D - Leu - L - Trp -

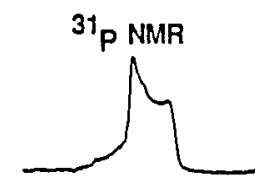

GRAMICIDIN A
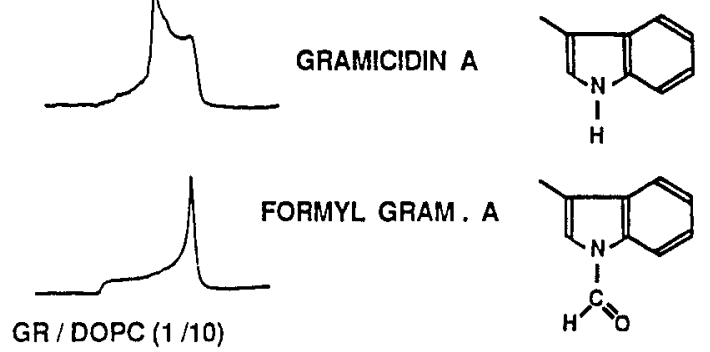

Fig. 8 3/P NMR spectra of dispersions of gramicidin and tryptophan formylated gramicidin with dioleoylphosphatidylcholine. For details see ref. 25

How can the preference for non bilayer structures and the role of tryptophans be understood? A likely explanation is the following. In general, when the hydrophobic part of a protein is much too short to fit into a lipid bilayer, this will result in protein aggregation $[1,25]$. Our hypothesis is that due to their strong preference for localization at the lipid/water interface [19], tryptophans may inhibit such aggregation. By organizing into non-bilayer structures, the favourable interaction of the tryptophans with the lipid/water interface can be optimally retained, while at the same time the hydrophobic mismatch is reduced or even completely removed. A model for such a peptide-induced non-bilayer structure is shown in Figure 9. 


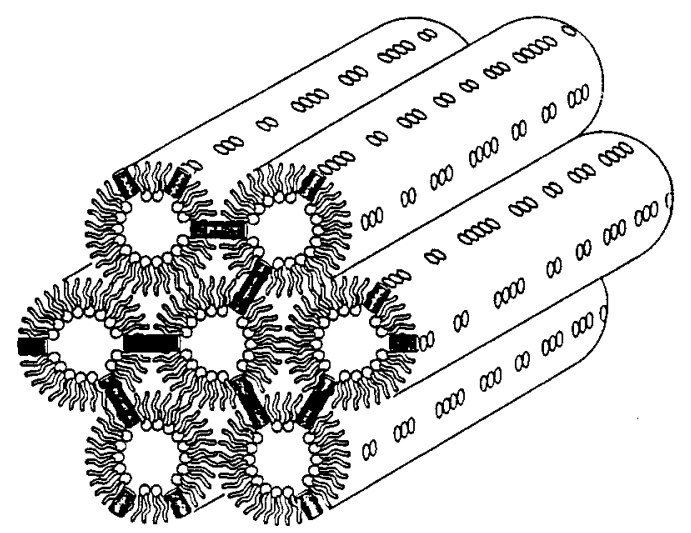

Fig. 9 Model of the WALP-induced HII phase. The peptides each span the distance between two tubes, such that all tryptophans are localized at the lipid/water interface. Since the peptides fill only the hydrophobic volume, the available area per head group will increase, relieving the steric constraints which normally will inhibit the formation of an HII phase for phosphatidylcholine. Due to the increased disorder of the acyl chains, the monolayer thickness of a lipid in an HII phase is much less than that of the same lipid in a bilayer. The monolayer thickness is smallest at the connections between adjacent water tubes, at which position the peptides are believed to be localized. Hence, a peptide that is too short to fit into a bilayer, can fit well into an HII phase formed by the same lipid. For details see ref. 18

\section{IMPLICATIONS FOR STRUCTURE AND FUNCTION OF PROTEINS AND LIPIDS IN MEMBRANES}

We have shown (1) that hydrophobic peptides can induce non-bilayer structures as a consequence of hydrophobic mismatch in phosphatidylcholine bilayers, (2) that the nature of these non-bilayer structures depends on the precise extent of mismatch, and (3) that the tryptophan residues are crucial for these effects. Thus, interfacially localized tryptophan residues are important determinants for consequences of hydrophobic mismatch.

\section{Possible implications for protein structure and function}

In membrane proteins tryptophans are frequently found at positions near the lipid/water interface $[15,16]$. The strong preference of these residues for a membrane/water interface has several implications. Under mismatch conditions, proteins are expected to have a high tendency to aggregate. However, the results presented here suggest that interfacially localized tryptophans will inhibit the aggregation process and therefore that other countereffects will be preferred. From the point of view of protein structure, a relevant possible adaptation will be a change in backbone conformation. Alternatively, when the membrane spanning part of the proteins is relatively long, the anchoring properties of interfacially localized tryptophans may cause the protein to tilt in the membrane. In multi membrane-spanning proteins it furthermore may be expected that the side to side packing of helices will be influenced by the tryptophans, i.e. the helices may have a tendency to associate with the tryptophan containing sides away from each other, and directed toward the lipids. Obviously, all adaptations described above can be expected to affect protein structure and therefore membrane protein activity. Further experiments will be focussed on elucidating the consequences of mismatch for protein backbone structure and tilt of the helices, and to find out to what extent other aromatic amino acids can function as membrane anchors.

\section{Possible implications for lipid structure and function}

The strong preference of tryptophans for a membrane/water interface also has several implications for lipid structure and function. As shown in the present study, when the membrane spanning part of the proteins is relatively short, the lipids can adopt non bilayer structures. The ability of lipids to adopt nonbilayer structures is essential for many functional properties of biological membranes and the amounts of bilayer and non-bilayer lipids appear strictly regulated [27, 28]. One function of non-bilayer lipids is believed to be their involvement in the local and transient formation of non bilayer structures, that are likely to occur during specific membrane processes, such as membrane fusion [10]. Another important 
function of these lipids may be to ensure a certain internal pressure in the membrane that might be required to keep membrane proteins in their functional state [13]. If the membrane-spanning part of tryptophan anchored proteins would be either too long or too short, it may thus be possible that the regulation of the amounts of bilayer and non-bilayer lipids becomes disturbed, thereby impairing membrane function. Alternatively, adaptations of lipid order and disorder in the membrane are possible as a consequence of mismatch, which would affect properties of the lipid bilayer. The extent to which these adaptations occur is currently being investigated.

\section{Acknowledgements}

This work was supported by the Netherlands Foundation for Chemical Research (SON), with financial aid from the Netherlands Organization for Scientific Research (NWO), by NIH Grant GM 34968 and by NATO Grant CRG 950357.

\section{References}

1. O.G. Mouritsen and M. Bloom. Biophys. J. 46, 141-153 (1984).

2. C. Montecucco, G.A. Smith, F. Dabbeni-sala, A. Johansson, Y.M. Galante and R. Bisson. FEBS Lett. 144, 145-148 (1982).

3. J. Riegler and H. Möhwald. Biophys. J. 49, 1111-1118 (1986).

4. M.S. Bretscher and S. Munro. Science 261, 1280-1281 (1993).

5. J.A. Killian, K.U. Prasad, D.W. Urry and B. De Kruijff. Biochim. Biophys. Actc 943, 535-540 (1989).

6. J.A. Killian, A.M.Ph. De Jong, J. Bijyelt, A.J. Verkleij and B. De Kruijff. EMBO J. 9, 815-819 (1990).

7. M.M. Sperotto and O.G. Mouritsen. Eur. Biophys. J. 22, 323-328 (1993).

8. Y-P. Zhang, R.N.A.H. Lewis, R.S. Hodges and R.N. McElhaney. Biochemistry 31, $11579-$ 11588 (1992).

9. B. Piknova, E. Perochon and J.-F. Tocanne. Eur. J. Biochem. 218, 385-396 (1993).

10. B. De Kruijff. Nature 329, 587-588 (1987).

11. J.N. Israelachvili, D.J. Mitchell and B.W. Ninham. J. Chem. Soc. Faraday Trans II 72, 1525 1568 (1976).

12. P.R. Cullis and B. De Kruijff. Biochim. Biophys. Acta 559, 399-420 (1979).

13. B. De Kruijff. Nature 386, 129-130 (1997).

14. C. Landolt-Marticorena, K.A. Williams, C.M. Deber and R.A.F. Reithmeier. J. Mol. Biol. 229 , 602-608 (1993).

15. H. Michel and J. Deisenhofer. Curr. Top. Membr. Transp. 36, 53-69 (1990).

16. C. Ostermeier, S. Iwata and H. Michel. Curr. Op. in Struct. Biol. 6, 460-466 (1996).

17. W.C. Wimley and S.H. White. Nat. Struct. Biol. 3, 842-848 (1996).

18. J.A. Killian, I. Salemink, M.R.R. De Planque, G. Lindblom, R.E. Koeppe II and D.V. Greathouse. Biochemistry 35, 1037-1045 (1996).

19. J. Seelig. Biochim. Biophys.Acta 515, 105-140 (1978).

20. G. Lindblom and L. Rilfors. Biochim. Biophys. Acta 988, 221-256 (1989).

21. M.M. Sperotto and O.G. Mouritsen Eur. Biophys. J. 16, 1-10 (1988).

22. C.J.A. Van Echteld, B. De Kruijff, A.J. Verkleij, J. Leunissen-Bijvelt and J. De Gier. Biochim. Biophys. Acta 692, 126-138 (1982).

23. J.A. Killian, K.U. Prasad, D.W. Urry and B. De Kruijff. Biochim. Biophys. Acta 978, 341-345 (1989).

24. F.J. Aranda, J.A. Killian and B. De Kruijff. Biochim. Biophys. Acta 901, 217-228 (1987).

25. J.A. Killian, J.W. Timmermans, S. Keur and B. De Kruijff. Biochim. Biophys. Acta 820, 154156 (1985).

26. D. Marsh. Mol. Membr. Biol. 12, 59-64 (1995).

27. A.G. Rietveld, M.C. Koorengevel and B. de Kruijff. EMBO J. 14, 5506-5513 (1995).

28. S. Morein, A.-S. Andersson, L. Rilfors and G. Lindblom. J. Biol. Chem. 271, 6801-6801 (1996). 